\title{
Magnetic Measurement of Alignment of Main LHC Dipoles and Associated Correctors
}

\author{
L. Bottura, M. Buzio, G. Deferne, P. Schnizer, P. Sievers, and N. Smirnov
}

\begin{abstract}
We discuss the method developed for the verification of alignment of magnetic elements contained in the LHC cryodipole cold mass during series tests at CERN. First, we outline motivations and requirements and then we focus on test strategy, equipment and procedures. Our goal is to express the magnetic field of the dipole and of its associated correctors w.r.t. the reference beam line, not accessible during cryogenic tests. To do so, we use traveling harmonic coil probes ("moles") that allow simultaneous measurement of the field and of the coil position. A laser tracker is used to relate these measurements to fiducials. In the dipole, the axis of the Quadrupole Configured Dipole (QCD) is used as an intermediate reference for the transfer. We provide details on the devices used for measurements in warm and cold conditions, some results from prototypes and pre-series dipoles and an assessment of the precision expected for the series tests.
\end{abstract}

Index Terms-Dipole correctors, fiducialization, LHC main lattice dipole, magnetic axis, quadrupole configured dipole.

\section{INTRODUCTION}

$\mathbf{T}$ HE LARGE Hadron Collider, to be built at CERN by 2005, includes 1232 two-in-one superconducting cryodipoles, providing a $8.36 \mathrm{~T}$ field in two $15 \mathrm{~m}$ long, $\varnothing 56 \mathrm{~mm}$ apertures [1]. The 0.97 MAturn, NbTi dipole coils are built into a cylindrical structure ("cold mass"), supported on three feet inside the cryostat and maintained at $1.9 \mathrm{~K}$. All of the dipole assemblies will include a sextupole corrector spool piece (MCS) at one end of each aperture, while one half will be additionally equipped with combined octupole-decapole correctors (MCDO) at the other end.

A pre-series run of 90 units has now started, with production ramping up to full scale in 2003 . The dipoles will be tested in industry and at CERN in both cryogenic and room-temperature conditions to be fully qualified for the machine. Extensive magnetic tests, in particular, will provide detailed information on field quality in terms of local and integrated strength, direction and field errors (i.e., higher-order multipoles) as a function of excitation level and powering history.

In this paper we focus on the aspects of magnetic tests at CERN related to the alignment of the dipoles. Broadly speaking, three main alignment issues need to be addressed. Firstly, the direction of the field must be measured w.r.t. the cryostat, so that the magnet can be correctly tilted in the tunnel. Secondly, multipoles must be expressed w.r.t. the theoretical beam line, so that their effect on the beam can be computed and the necessary corrective actions taken. Finally, the alignment of the in-built correctors w.r.t. beam line must be accurately verified,

Manuscript received September 24, 2001.

The authors are with CERN, European Center for Nuclear Research, CH-1211 Geneva 23, Switzerland (e-mail: marco.buzio@cern.ch).

Publisher Item Identifier S 1051-8223(02)04264-1.

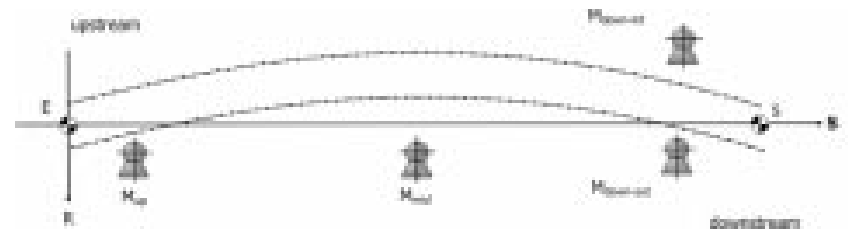

Fig. 1. The standard MAD dipole right-handed coordinate frame $\{R, S, T\}$ (seen from above). The $(R, S)$ plane is the defined by the two theoretical beam line arcs; the $S$ axis is defined by the entry and exit points $E$ and $S$. The four $M_{i}$ points represent cryostat fiducials (optical targets).

TABLE I

STANDARD DEVIATION OF RANDOM ALIGNMENT ERRORS (AZIMUTHAL)

\begin{tabular}{lccc}
\hline specifications for: & whole accelerator individual magnets \\
\hline b3 corrector wrt dipole & 0.18 & 0.5 & $\mathrm{~mm}$ \\
b4 corrector wrt dipole & 1.60 & 0.5 & $\mathrm{~mm}$ \\
b5 corrector wrt dipole & 0.28 & 0.5 & $\mathrm{~mm}$ \\
dipole w.r.t. DO & 0.29 & 1.0 & $\mathrm{~mm}$ \\
\hline
\end{tabular}

to avoid that the corrective action generates spurious multipoles by feed-down [2].

\section{Overall StRAtegy For AlignMent Measurements}

\section{A. Requirements for Alignment Verification}

The integrated field generated by an accelerator magnet can be considered as bi-dimensional and can be described in terms of its multipole coefficients, referred to a given center, as a function of the excitation. Machine tolerances are expressed in terms of the position of such center w.r.t. the design orbit (DO). The ultimate goal of our alignment tests is therefore the transfer of magnetic measurements, including dipole harmonics and corrector axes, onto the DO.

The DO for LHC bending magnets is defined as the leastsquares best fit of two co-planar arcs of circle of radius $R=$ $2812.36 \mathrm{~m}, 194 \mathrm{~mm}$ apart, to the mechanical axes of the cold bores. The reference system $(R, S, T)$ used by the MAD code for beam optics [3] is defined on the basis of these arcs, as shown in Fig. 1. This frame is materialized on the cryostat by four fiducials $M_{i}$, realized with standard optical targets. While three fiducials are in principle sufficient to describe completely the 3-D position of a rigid body, a fourth was added in the middle of the magnet (along with an additional support jack) to measure and control magnet sag.

A summary of dipole alignment requirements and specifications is given in Table I [4]. These figures, which represent standard deviation of random errors, should be considered as an upper bound for the accuracy required for our alignment tests. Systematic measurement errors cannot yet be assessed and have not been included in this analysis. 


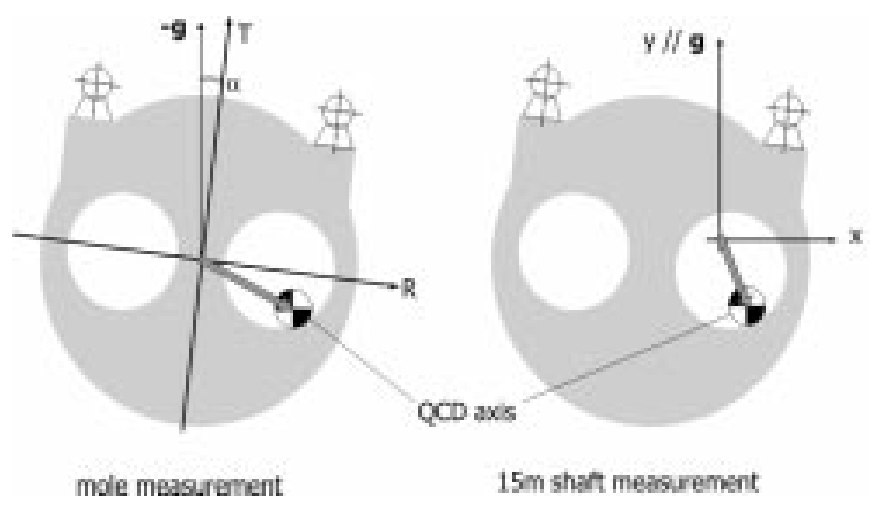

Fig. 2. Schematic of multipole transfer to the $(R, S, T)$ frame. Multipoles and axis are measured first with the TRS system w.r.t. the $\{x, y\}$ coil reference frame (right). The axis is then measured again with a mole w.r.t. the $\{R, T\}$ frame (left), and is used as an intermediate reference for the transfer. Both frames are first oriented to the gravity $(g)$.

\section{B. Overview of Alignment Tests}

During cold series tests at CERN, multipoles will be obtained as the harmonic components of the flux picked up by the Twin Rotating Coil System (TRS). This includes two 15-m long segmented ceramic shafts carrying each $13 \times 1.15 \mathrm{~m}$ long dipolecompensated coils. The system, working at room temperature inside a heated pipe passing through the cold bores (i.e., an anticryostat), is able to measure the field all along the magnet within a few seconds [5].

Measured multipoles are naturally expressed in a frame of reference centered on the coil rotation axis and aligned to the gravity. The position of the center in the cross-section is affected by an uncertainty of the order of a millimeter, due to the flexibility in bending of the anticryostat on its supports inside the cold bore. Such an error cannot be recovered by direct measurements, since the coil shaft is not visually accessible during the tests. Therefore, the centering must be carried out separately with traveling probes.

The transfer of measured multipoles to the $(R, S, T)$ frame is done in several steps, which are essentially similar for both dipoles and correctors (Fig. 2). First, a high-field compatible, rotating-coil traveling probe ("cold mole") is used to measure simultaneously the magnetic axis and, via optical detection, the coil rotation center w.r.t. the fiducials. The magnetic axis measured with this tool is then used to transfer multipoles (measured with the TRS in a wide range of dynamic conditions) to the fiducials. Finally, fiducialization data (i.e., the $(R, S, T)$ coordinates of the four magnet fiducials measured at room temperature and extrapolated to cryogenic conditions [6]) are used to transfer the magnetic measurements to the reference beam line.

At present, this procedure is foreseen for the majority of preseries dipoles. If tolerances will be consistently met, one can realistically envisage that only a statistically relevant sample of the series (say, 10\%) will be measured with a cold mole, due to time and cost constraints. If necessary, however, an existing room-temperature version of the probe ("warm mole") will be used more systematically to establish and apply a warm/cold correlation.
TABLE II

SUMMARY OF RANDOM ANGLE ERRORS

\begin{tabular}{lccc}
\hline Aagle error component & Local & Average & \\
\hline Field angle wrt coil (cal. bench 9 0.5T) & 0.02 & n.a. & mrad \\
Field angle wrt coil (dipole 08.36T) & 0.01 & n.a. & mrad \\
Calibration procedure (reference bilt) & 0.10 & 0.10 & mrad \\
Fieid angle wrt gravity & 0.15 & 0.15 & mrad \\
Fiducials wrt gravity & 0.03 & 0.03 & mrad \\
Cold mass wrt fiducials & 0.15 & 0.15 & mrad \\
\hline Total error & $\mathbf{0 . 2 5}$ & $\mathbf{0 . 0 8}$ & $\mathrm{mrad}$ \\
\hline Magnet specification & 3 & 1 & mrad \\
LHC requirement & n.a. & 1 & mrad \\
\hline
\end{tabular}

\section{MEASUREMENT OF Field DireCtion}

\section{A. Measurement Procedure and Calibration}

The orientation of rotating coil measurements is given by the coil tilt angle at the time when flux integration is triggered (usually by an angular encoder). In our TRS system this angle is affected by assembly tolerances larger than the precision required and a magnetic calibration is therefore necessary.

Angle calibration is carried out on a dedicated scanning bench, equipped with a motorized, $0.5 \mathrm{~T}$ resistive dipole with a field ripple $<2 \cdot 10^{-4}$. The direction of field of the magnet w.r.t. gravity is calibrated independently with a precision of $0.02 \mathrm{mrad}$. On this bench, measurements are done with the TRS at opposite current polarity and averaged to cancel residual magnetization effects. The difference between the true and measured field angle is used later to correct measurements done inside cryodipoles.

A laser tracker is used to link cryodipole fiducials to the vertical, and fiducialization data can then be used to refer field angle to the mean plane of the dipole in cold conditions (at room temperature, the cold mass is optically accessible and therefore a direct measurement can be done). A breakdown of the various sources of random errors affecting the final accuracy is given in Table II.

Systematic errors will be estimated on a statistical basis by comparison with measurements done with independent systems. Preliminary results obtained with a stretched wire system [7], taking into account the curvature of the magnet, agree to better than $0.2 \mathrm{mrad}$ in cold conditions. Warm field angle measurements done with low-field moles generally agree within $0.5 \mathrm{mrad}$.

\section{B. Results of Prototype and Pre-Series Dipoles}

Measurements of prototypes and of the first pre-series dipoles show that the field direction is usually well within the acceptable tolerances. Most magnets exhibit a total and local twist not exceeding $3 \mathrm{mrad}$ in both cold and warm conditions, while the parallelism between apertures is generally better than $1.0 \mathrm{mrad}$.

The average direction change with excitation level has been found to be smaller than $0.1 \mathrm{mrad}$ up to $12 \mathrm{kA}$. The effect of the elevation of the central jack has also been measured. Due to the curved shape of the cold mass, the cold mass rotates by up to $0.05 \mathrm{mrad} / \mathrm{mm}$ at the nominal $11.85 \mathrm{kA}$ current (up to $0.07 \mathrm{mrad} / \mathrm{mm}$ at $5 \mathrm{kA}$ ).

As an example, Fig. 3 shows the field direction as a function of longitudinal position for the one of the pre-series magnets. 


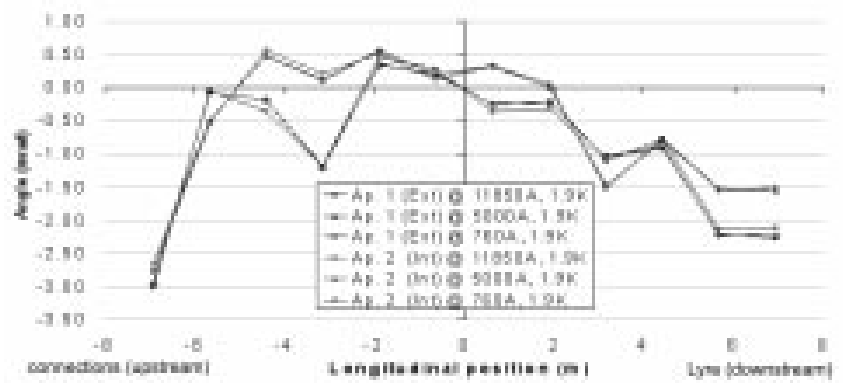

Fig. 3. Field angle of pre-series dipole Noell 2 w.r.t. the mean plane of the cold mass $(R, S)$ for both apertures at injection, nominal and $5 \mathrm{kA}$ current.

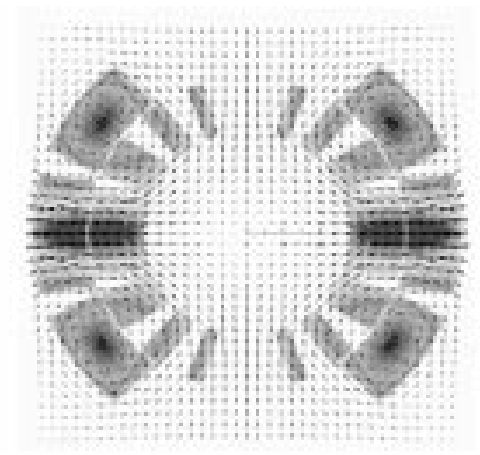

Fig. 4. Quadrupole-configured dipole field map, computed with ROXIE.

\section{QUADRUPOLE-CONFIGURED DiPOLE AXIS}

Dipole harmonics measured with the TRS can be transferred to the frame of the rotating coil of the mole (and, later, to the fiducial frame) by using a suitable magnetic axis as a common reference. The preferred reference for tests at CERN is the axis of the so-called Quadrupole-Configured Dipole (QCD, or "ugly quadrupole"), obtained by feeding currents of opposite polarity into the top and bottom half-coils through the voltage taps (Fig. 4). Center coordinates are computed from linear feed-down (assuming that the measured dipole is purely due to the offset between coil rotation axis and magnetic axis):

$$
\begin{aligned}
& \frac{\Delta x_{Q C D}+i \Delta y_{Q C D}}{r_{R E F}} \approx-\frac{B_{1}+i A_{1}}{B_{2}+i A_{2}}, \\
& r_{R E F}=17 \mathrm{~mm}
\end{aligned}
$$

The QCD axis offers several advantages over other magnetic center definitions, such as those based on the elimination of unallowed dipole harmonics (e.g., $b_{10}$ or $b_{16}$, generated mainly by feed-down from a strong $b_{11}$ and $b_{17}$ due to the LHC cable geometry $)^{1}$ :

1) it is strictly related to the current centroid, and therefore stably linked to the shape of the cold mass as a whole;

2) it lies closer than $0.1 \mathrm{~mm}$ to the mechanical axis, therefore it is suitable as a warm reference for corrector alignment (to be confirmed statistically);

3 ) it can be measured with as little as $0.5 \mathrm{~A}$ with a resolution of $8 \mu \mathrm{m}$, compared to about $50 \mu \mathrm{m}$ for the $b_{10}$ axis.

4) it can be measured easily with d.c. excitation, with the same equipment used for dipole harmonics.

${ }^{1}$ Note that the axes of low-order harmonics cannot be defined, as they move with superconductor hysteresis and other dynamic effects.

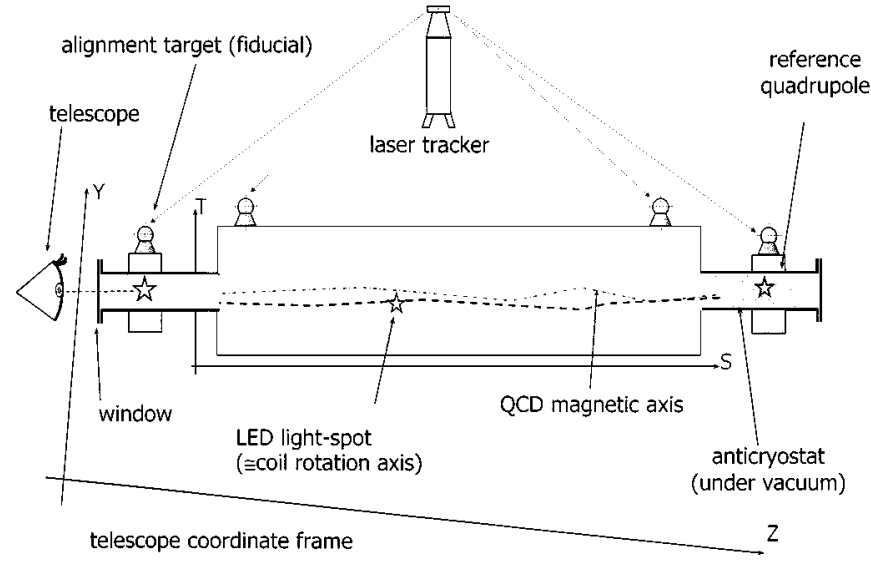

Fig. 5. Overall scheme of the magnetic axis fiducialization procedure, carried out with a high-field mole at cryogenic temperature.

Current has to be injected from two separate sources per aperture, which must be accurate to about $10^{-3}$ to ensure vertical field symmetry. The powering scheme must also be compatible with the existing protection system installed at the voltage taps. The two apertures must be fed with equal and opposite currents to compensate cross-talk effects leading to an apparent horizontal axis shift of about $0.5 \mathrm{~mm}$ for a $100 \%$ imbalance. Measurements have to be done at both polarities and averaged to cancel out residual magnetization effects.

Finally, the transfer between TRS and mole measurements must take into account a systematic radial axis shift due to the sagitta of a coil of length $\ell_{C}$ inside the beam pipe, given by $\Delta x \approx 1 / 8 \ell_{C}^{2} R^{-1}$. This shift averages to approximately $(2 / 3) \Delta x$, i.e., $17 \mu \mathrm{m}$ for the moles and $39 \mu \mathrm{m}$ for the $15-\mathrm{m}$ shaft system.

\section{Axis Fiducialization With Traveling Probes}

\section{A. High-Field Probe ("Cold Mole")}

The cold mole is a travelling harmonic coil probe designed to measure simultaneously magnetic field and position of the search coil on the cryogenic test bench. One unit is now being built at Fraunhofer IPT and is expected to be operational by end 2001.

In the cold mole, the coil rotation axis is indicated by a LED source and imaged by a telescope with a CCD camera system, able to calculate the position of the light spot relative to the pointing direction of the telescope with a precision better than $30 \mu \mathrm{m}$. The probe $(\varnothing 36 \mathrm{~mm})$ runs inside an anticryostat evacuated to eliminate light path distortions due to thermal gradients. The magnetic flux is detected with the modular acquisition system used for all tests at CERN, based on analog compensation and voltage-to-frequency digital integration.

The axis fiducialization procedure consists in the transfer of the coordinates of the QCD magnetic axis to the reference frame of the fiducials. The axis is measured as a function of longitudinal position at $N$ discrete steps, with typically $N=20$. As shown in Fig. 5, the transfer is carried out with the help of two reference quadrupoles at both ends of the magnet, linking measurements done inside the magnet apertures to those done outside. At each longitudinal position, the position $\mathbf{C}_{i}$ of the light-spot is measured with the telescope, while the axis coordinates $\left(\mathbf{Q}_{i}-\mathbf{C}_{i}\right)$ are computed by feed-down. The position $\mathbf{F}_{i}$ 


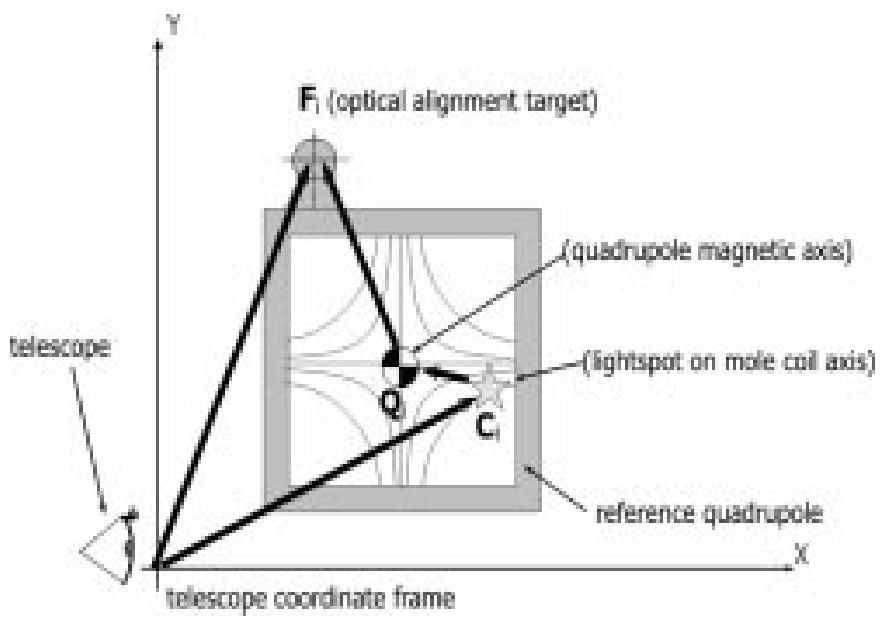

Fig. 6. Measurement of reference quadrupole fiducials in the telescope coordinate frame.

TABLE III

ESTIMATED RANDOM ERRORS IN MAGNETIC AXIS MEASUREMENT

\begin{tabular}{lccc}
\hline Error source & MB & MCS,MCDO \\
\hline Magnetic axis to coil axis & 40 & 10 & $\mu \mathrm{m}$ \\
Coil axis to telescope & 33 & 32 & $\mu \mathrm{m}$ \\
Reference fiducials to coil axis ( 2) & 46 & 46 & $\mu \mathrm{m}$ \\
Coil axis to telescope at references( 2) & 65 & 65 & $\mu \mathrm{m}$ \\
\hline Reference fiducials to magnet fiducials & 115 & 115 & $\mu \mathrm{m}$ \\
\hline Total (RMS) & $\mathbf{1 6 7}$ & $\mathbf{1 6 2}$ & $\mu \mathrm{m}$ \\
\hline
\end{tabular}

of the reference quadrupole fiducials in the telescope frame can be obtained from (Fig. 6):

$$
\mathbf{F}_{i}=\mathbf{C}_{i}+\left(\mathbf{F}_{i}-\mathbf{Q}_{i}\right)+\left(\mathbf{Q}_{i}-\mathbf{C}_{i}\right), \quad \text { with } i=0, N+1
$$

where the vector $\left(\mathbf{F}_{i}-\mathbf{Q}_{i}\right)$ is measured on a dedicated calibration bench. A laser tracker is used to measure the 3-D coordinates of the fiducials on the magnet $\mathbf{M}_{i}$ and on the reference quadrupoles $\mathbf{F}_{i}$. The transformation between the two reference frames, provided both vertical axes are referred to gravity, can then be obtained simply by superposing $\mathbf{F}_{0}$ and $\mathbf{F}_{N+1}$. By using this procedure, dipole multipoles and corrector axes can be expressed w.r.t. the cryostat and used directly to align the magnet in the tunnel. Random errors, estimated on the basis of preliminary tests, are given in Table III.

In addition, the magnetic multipoles and axis can be given directly w.r.t. the mechanical axis of the cold bore, inside which the probe is mechanically centered, although with a larger uncertainty about $0.2-0.5 \mathrm{~mm}$.

Note that, according to the current hardware baseline, the position of the central cryostat support jack at the time of cold tests will not necessarily be the same as in the tunnel. This may lead to a vertical deformation of the dipole axis of the order of a few $\mathrm{mm}$. To correct for this deformation, the possibility of parameterizing both warm and cold QCD axis measurements versus jack elevation is currently being explored.

\section{B. Low-Field Probe ("Warm Mole")}

This is a simpler version of the probe described above, described in detail in [8] and designed to work at room temperature in free air directly inside the beam pipe of the magnet. One unit

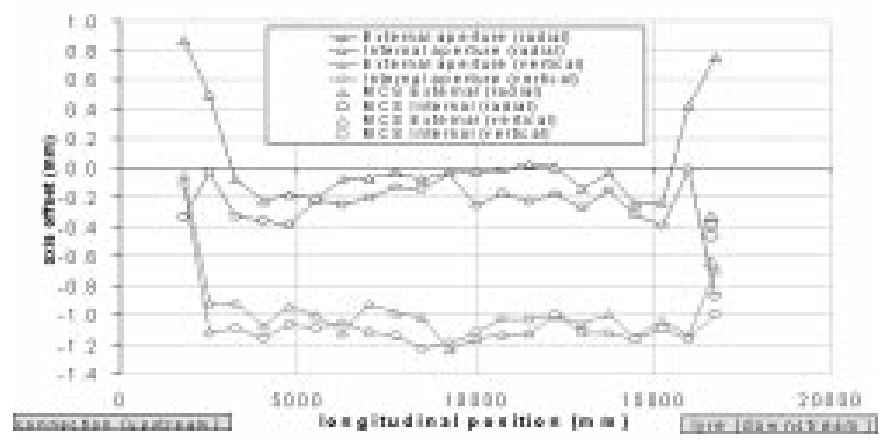

Fig. 7. QCD axis of Alstom 2 w.r.t. coil rotation axis at room temperature.

is operational since 1999 , while two more units are now being procured.

During pre-series tests, the warm mole is foreseen to be used at the beginning of each campaign to establish an initial reference for the QCD magnetic axis and to carry out magnetic quality control spot-checks, e.g., to detect changes in the magnet due to transportation from the manufacturer's site. Most importantly, however, we plan to use this instrument at the end of each cold test to fiducialize the QCD axis making use of an established warm/cold correlation, and to verify the alignment of the dipole axis relative to correctors (which can only be powered after the end of the standard cold tests). As an example, Fig. 7 shows warm mole results for one of the pre-series dipoles tested so far.

\section{CONCLUSIONS}

The procedures we outlined here are well suited to check machine critical parameters, such as corrector alignment, by directly accessing magnetic field parameters without relying on mechanical assumptions or extrapolations. Mole tests in cold magnets are uniquely capable to assess the relative alignment between correctors and dipole, as well as the position of the cold mass relative to fiducials in realistic cryogenic conditions. The balance of cold and warm tests necessary to fulfill requirements will be determined with more confidence on the basis of the results obtained from pre-series magnets tests, which are now under way.

\section{REFERENCES}

[1] M. Modena et al., "Manufacture and performance of the LHC main dipole final prototypes," in 7th EPAC, Vienna, Austria, June, 26-30, 2000.

[2] W. Scandale et al., "Geometric and magnetic axis of the LHC dipole," in PAC 2001, Chicago, IL, June 18-22, 2001.

[3] F. C. Iselin, J. M. Jowett, J. Pancin, and A. Adelmann, "AMAD version 9," in 7th EPAC, Vienna, Austria, June, 26-30, 2000.

[4] A. Verdier and E. Wildner, "Feed-down effect in dipole alignment," PAC 2001, June 18-22, 2001.

[5] L. Bottura and M. Buzio et al., "Twin rotating coils for cold magnetic measurements of $15 \mathrm{~m}$ long LHC dipoles," IEEE Trans. Appl. Superconduct., vol. 10, no. 1, pp. 1422-1426, 2000.

[6] D. Missiaen, "Fiducialization of LHC cryo-dipole magnets,", CERN-LHC Engineering Specification LHC-GI-ES-0002.

[7] J. di Marco et al., "Field alignment of quadrupole magnets for the LHC interaction regions," IEEE Trans. Appl. Superconduct., vol. 10, no. 1, pp. 127-130, 2000.

[8] L. Bottura and M. Buzio, et al., "A mole for warm magnetic and optical measurements of LHC dipoles," IEEE Trans. Appl. Superconduct., vol. 10, no. 1, pp. 1454-1457, 2000. 